\title{
Operational Communications: Adapting a Hospital Daily Check-In (DCl) for Expanded Use During Emergency Events
}

Kimberly Bailey, MA; Kyle Williford, BA (1); Patrick Cawley, MD, MBA, MHM; Matthew J. Wain, MAS; Kathy Lehman-Huskamp, MD

\section{ABSTRACT}

Low-frequency, high-acuity emergency events can and do occur within health care settings. Having a strong sense of daily situational and operational awareness is the first step in responding to any emergency event. To maintain high reliability, hospital leaders and staff must understand the full impact to the organization as the emergency event evolves. The Medical University of South Carolina health system has implemented the common practice of a daily operations safety briefing, called the Daily Check-In, to communicate any issues that could impact the operational ability of the hospitals within the enterprise, or any other associated resources during a disaster or emergency. Throughout various emergency events, including extreme weather, the Daily Check-In has evolved as a standard process for use during emergencies that is open to all staff and uses highly reliable systems.

Key Words: communication, decision making, disaster planning, emergency preparedness, floods

$\mathrm{H}$ igh reliability organizations (HROs) are guided by a set of 5 principles that support the goal of exceptional safety: preoccupation with failure, reluctance to simplify, sensitivity to operations, commitment to resilience, and deference to expertise. ${ }^{1}$ As an $\mathrm{HRO}$, the health system associated with the Medical University of South Carolina (MUSC Health) works continuously to refine its processes in pursuit of unfailing safety. The implementation of an HRO mindset at MUSC Health is centered around an emphasis on creating a culture of safety through a model based on meta-leadership called optimal accountability, which comprises 3 distinct accountability levels where leaders are vertically accountable through an organizational hierarchy, peers are horizontally accountable to each other, and individuals are expected to be intrinsically accountable. One of the ways MUSC Health maintains optimal accountability is through robust information sharing and communication links, including a daily operations and safety briefing called the Daily Check-In (DCI). The DCI aligns the collective mindset of MUSC Health with sensitivity to operations and facilitates optimal accountability using status reporting and metrics to identify and address operational issues as they occur.

The main tenet of an $\mathrm{HRO}$ is to function reliably, despite adverse or dangerous conditions. As a hospital system, MUSC Health has an obligation to perform reliably, safely, and with quality, despite external conditions. Situated at sea level and surrounded by coastal water, MUSC Health emergency management planning occupies an important role in maintaining high reliability under a range of environmental emergencies that could risk operational viability, including regular flooding and severe tropical weather. Part of MUSC Health's emergency management planning includes a Hospital Emergency Operations Center (HEOC) that activates and coordinates resources to ensure uninterrupted patient care. A moment of need during an extraordinary flood event in 2015 led the HEOC to adapt the health system's existing DCI as an emergency management tool, sparking a shift in how emergency communications and operations are conducted at MUSC Health. Through subsequent scenarios in which the DCI was deployed as part of the MUSC Health HEOC, further refinement and improvement of the tools and techniques used to conduct the briefing have been developed.

\section{OVERVIEW OF DCI}

The DCI has been widely adopted as a best practice of HROs in many industries, including health care. The practice has, in fact, been adopted in several other health care organizations, including Advocate Health Care, ${ }^{2}$ Community Hospital North of Community Health Network (CHN), Helen DeVos Children's Hospital of Spectrum Health (HDVCH), Virginia 
Commonwealth University Health System (VCUHS), Wyoming Medical Center (WMC), and Yakima Valley Memorial Hospital (YVMH). ${ }^{3}$ Though DCI is difficult to measure in terms of direct impact on quality of care, many organizations cite reduced serious safety event rates since instituting DCI, and VCUHS and CHN both reported positive perception scores from leaders relating to the impact of DCI. ${ }^{3}$ As HROs, hospitals can maintain sensitivity to operations using DCI as a method of recognizing issues early through multilevel participation and open communication. ${ }^{4}$

Its fundamental purpose is to focus every area of the organization on operations and safety at the beginning of each day, and to provide an opportunity for coordination between clinical care team members, facility managers, and administration. ${ }^{5}$ The DCI captures the 24-hour status of the various organizational groups involved in safe and reliable operations and maintains sensitivity to operations through a state of organizational mindfulness. By holding a DCI call every day, MUSC Health leadership maintains awareness of issues that could impact the ability to respond to events. For example, knowing how many patients are in the hospital at any given time will help with resource requests for transportation and allocation of those resources if the hospital must be evacuated emergently. Under normal operations, the DCI is accessible to all members of the organization and includes reports from 28 areas on quality, bed flow, shared services, service lines, and the environment of care. The DCI is facilitated by an Administrator On-Call (AOC) who tracks and follows up on actions and issues with leaders responsible for the various reporting areas. The responsible leader of a reporting area is accountable for relaying information and coordinating action items in the DCI. Because it involves so many functional areas of the organization, the DCI allows MUSC Health to sustain operational responsiveness day to day, without the constraints of siloed reporting and more hierarchical communication methods. As a daily information brief and a means of coordinating efforts to identify and resolve issues, the DCI has become a vital tool for the organization.

\section{EMERGENCY OPERATIONS DCI 1000-Year Flood (2015)}

Though an established practice during normal operations, the first deployment of the DCI by the MUSC Health HEOC came during 1 of the most extreme rain events ever documented in the state of South Carolina. In October 2015, the churning Charleston harbor broke over the sea wall and water gushed from storm drains throughout the city as nearly 2 feet of water inundated the area over the course of 4 days. Flooding is typical around MUSC Health's downtown campus, with nuisance flooding occurring at an average of 23.3 days per year (2007-2013). ${ }^{6}$ However, the severity of "1000-year flood" - so called because it statistically had a 1 in 1000 chance of happening in any given year - was so great that a new approach to coordinated emergency communication was necessary to maintain sensitivity to operations.

As an HRO, MUSC Health must perform "flawless communication and coordination to maintain the situation awareness (SA) required to manage these rapidly changing and complex events." To provide group SA to all leadership levels in preparation for the 1000-year flood, the HEOC was initialized using $\mathrm{DCI}$ as the primary means of operational communication for the first time. The DCI during this emergency event represented a conscious effort to continue high-reliability practices regardless of circumstances. Despite the impact to the organization, the continuation of an already reliable practice allowed staff to operate with the enhanced awareness and communication required by high-reliability teams. As the 1000-year flood unfolded, groups within MUSC Health, such as the Pediatrics Emergency Department, began to place the DCI on speaker phone for staff, deploying the meeting as a unit huddle. Rather than matriculate information through leadership to staff, all levels of the organization could receive unfiltered situational awareness concerning operations and contingencies in real-time, resulting in time saved and increased transparency. Though high-level direction must follow a chain of command in the form of top-down resolutions, organizational awareness among all levels of staff provides greater understanding of why and how decisions are made, and the DCI provides every care team member the same message.

The DCI is not intended to be directive, and rather provides the tools and information sharing necessary for unit and department-level leadership to make decisions and take action. As frontline staff, they are most in tune to issues that directly impact their teams and are therefore better equipped to share information and direct resources under their control. Optimal accountability, like meta-leadership, relies on connectivity for individuals to communicate freely, ${ }^{8}$ and frontline staff are better positioned to respond to and understand issues when information is more readily available. Though a minor change to the way the DCI had been used, nurse leaders facilitating the call for the entire unit were able to connect frontline staff directly with leaders and other groups within the organization allowing the entire staff to be aware of the same information at the same time, breaking down the restrictive aspects of strictly hierarchical information dissemination and improving accountability and efficiency.

Frequency of communication and familiarity with emergency management processes can have enormous impacts on organizational operations during an emergency. ${ }^{9}$ While preparedness through training and exercises focuses staff on response readiness, the coordination and management of resources in an emergency can be seamlessly performed by the DCI. The reporting staff are familiar with the format and the pertinent information that must be provided, normalizing the transition from an everyday process into an emergency process. Using the 
same basic reporting template as a daily operations DCI, the HEOC can appropriate the DCI during an emergency without requiring rehearsal or training of staff. The DCI is modified to suit the unique conditions of every event, but the basic function and process change little during an emergency. In all instances, the reporting template includes different functional areas of the health system and their responsible parties, a look back into events since the last DCI, a look forward at potential issues, and a follow-up of action items and issues identified previously. All reporting at the DCI is documented and posted for enterprise-wide review on MUSC Health intranet sites. During an emergency, information from each DCI is reviewed by the AOCs and operational leadership for relevance to all care team members. Essential information is parsed into official messaging via e-mail, websites, and other forms of internal communication. Serving as a source of information that matriculates throughout the organization, an emergency DCI occurs every 4 hours or more frequently as conditions warrant. Further summarizing the conference call in followup messages maintains communication control while still providing transparency. Increased frequency ensures that the most current reports and immediate issues are reviewed as soon as they occur and maximizes operational sensitivity.

Following every major emergency event since Hurricane Matthew in October 2016, a voluntary, online, after-action survey has been used to collect data on employee perceptions of the organization's handling of events. The survey asks respondents to assess the effectiveness of the organization and its processes and tools during an emergency. After-action surveys for emergency incidents that have occurred since the institution of DCI in such situations indicate communication as a recurring positive element of operations. The surveys offer leaders the opportunity to collect feedback from staff using 3 simple response criteria: what worked well, what did not work well, and what could be done to improve the organizational response to future events.

Each threat since its initial use has offered an opportunity for refinement and testing of the operations and safety briefing model, as lessons learned and after-action feedback have indicated new and better ways of deploying the DCI. The HEOC DCI and its use as a unit huddle during emergencies were noted in the after-action report as best practices, and subsequent events saw the HEOC DCI adopted more widely for unit huddles. The positive after-action reports from the 1000 -year flood led to adjustments in the Emergency Management Plan and the incorporation of the DCI in future events. As significant natural disasters continued to threaten MUSC Health in coming years, the use of DCI would continue to evolve.

\section{Winter Storm (2018)}

Commonly occurring events, like nuisance flooding, allow emergency management procedures to become refined and well-rehearsed, but a winter storm in 2018 brought some unique and unanticipated challenges to MUSC Health. Until that time, all of the major incident responses using DCI had been for water-related events (flooding, hurricanes, etc.). In January 2018, a truly unusual occurrence impacted the region and threatened operations at MUSC Health. The winter storm of 2018 brought 5 to 7 inches of snow accumulation to an ill-equipped area with annual accumulation averages of zero. Snowfall immobilized the surrounding areas, causing travel and logistical problems for thousands of people. Secondary and tertiary roads were unplowed and remained so for days. With little to no salting of local roads, the snow soon turned to thick sheets of ice, causing a lasting hazard to commuters and emergency vehicles. Weather alerts for the area were incorporated into DCI's leading up to the event and allowed for assessing the preparedness of the organization and facilities, staff planning, and requesting emergency supplies. As a wintry mix began to fall, the HEOC was formally established and the DCI was used to coordinate efforts to test generators, locate and secure areas with poor heating, and relay parking information.

As part of the emergency management protocol, a designated primary response team is expected and prepared to remain at MUSC Health for the duration of an anticipated event (Team A), with relief staff offsite until conditions permit their return (Team B). Hurricanes approaching the Charleston area sometimes prompt evacuation orders from the state government, and, in those instances, the relief team may evacuate, ensuring the safety of non-essential personnel and limiting the number of participants in the DCI to the essential staff remaining in the area. Unanticipated impact levels from events like snow and ice storms mobilize a modified primary response team of staff who are already onsite. When an event affects transportation routes to and from MUSC Health, the relief team stands by until conditions improve. Official direction is cascaded to teams from direct supervisors and other means, but the reliance on the DCI as a real-time source of information had become common throughout the organization at the time of the winter storm. With relief staff confined to their homes and seeking greater situational awareness, the demand on the telephone trunk line carrying the DCI quickly exceeded its capacity of 200 external callers. Though the HEOC DCI helped MUSC Health maintain uninterrupted clinical care through identifying issues and coordinating resources, the inadequate trunk line prevented some staff from joining the call to listen or report statuses, presenting a potential failure in maintaining high reliability. As part of the greater HRO effort of MUSC Health, the DCI served the clinical staff of the hospital as a communication tool that facilitated greater awareness among staff.

Limitations of the technology used for the DCI in the 2018 winter storm led to an analysis of its vulnerabilities, which determined that, during a phone outage, the DCI call line would not function and was not a reliable means of communication. To overcome this deficiency, MUSC Health reviewed 
alternative systems that could provide reliably accessible communication for high-volume calls. Cisco Webex, already in use at MUSC Health for online collaboration, was selected as an alternative system on which to conduct the DCI. Webex is a cloud-based collaboration service that includes capabilities for voice over Internet protocol (VOIP), online, and video conferencing. The Webex Audio service allows MUSC Health to include 1000 participants, internal or external callers, in the DCI, thus greatly reducing the likelihood of a responsible leader being unable to report their status. As an existing asset, additional training and costs of using Webex were not required, and the system has worked without issue since being implemented for DCI calls. Though MUSC Health uses Webex, similar products could be used in any organization, including GoToMeeting, Skype, Zoom, among others.

\section{CONCLUSION}

The continued development of the MUSC Health DCI as a tool for conducting emergency management communications has resulted in a more transparent and operationally sensitive organization. With the aim of high reliability, the DCI supports familiarity with the emergency management communication process, efficiency of information sharing, and optimal accountability. The use of the DCI as a method of emergency management communication has evolved as different events have presented unique challenges. Through continuous improvement, the DCI as a function of the HEOC effectively streamlines complex communications and actions to ensure that unanticipated events are handled with resilience. From sharing the call during unit huddles, to ensuring accessibility with technology, the DCI has changed the way the MUSC Health HEOC is conducted and will continue to shape and inform methods in the future.

\section{About the Authors}

Medical University of South Carolina Health, Charleston, South Carolina.

Correspondence and reprint requests to Kyle Williford, 171 Ashley Ave., Suite 419, MSC 403, Charleston, SC 29425 (e-mail: willifok@musc.edu).

\section{REFERENCES}

1. Weick KE, Sutcliffe KM. Managing the unexpected: sustained performance in a complex world. Somerset, United States: John Wiley \& Sons; 2015.

2. Sikka R, Kovich K, Sacks L. How every hospital should start the day. Harvard Business Review. 2014. https://hbr.org/2014/12/how-everyhospital-should-start-the-day. Accessed August 14, 2019.

3. Stockmeier C, Clapper C. Daily check-in for safety: from best practice to common practice. Patient Saf Qual Healthc. 2011;8:30-31, 34-36.

4. Chassin MR, Loeb JM. High-reliability health care: getting there from here. Milbank Q. 2013;91(3):459-490.

5. Hines S, Luna K, et al. Becoming a high reliability organization: operational advice for hospital leaders. (Prepared by the Lewin Group under Contract No. 290-04-0011.) AHRQ Publication No. 08-0022. Rockville, MD: Agency for Healthcare Research and Quality; 2008.

6. Sweet W, Park J, Marra J, et al. Sea level rise and nuisance flood frequency changes around the United States. NOAA Technical Report. 2014; doi:10. $13140 / 2.1 .3900 .2887$

7. Autrey P, Moss J. High-reliability teams and situation awareness: implementing a hospital emergency incident command system. J Nurs Admin. 2006;36(2):67-72.

8. Farra SL, Miller ET, Gneuhs M, et al. Disaster management: communication up, across, and down. Nurs Manage. 2017;48(7):51-54.

9. Carley S, Mackway-Jones K. Major incident medical management and support: the practical approach in the hospital. Chichester, United Kingdom: John Wiley \& Sons; 2005. 